\title{
Risk factors for frequent hospital readmissions for acute exacerbations of COPD
}

\author{
Gaude Gajanan*, Hattiholi Jyothi, Chaudhury Alisha \\ Department of Pulmonary Medicine, KLE University's Jawaharlal Nehru Medical College, Belgaum, Karnataka, INDIA
}

Email address:

gsgaude922@gmail.com (G. Gajanan)

To cite this article:

Gaude Gajanan, Hattiholi Jyothi, Chaudhury Alisha. Risk Factors for Frequent Hospital Readmissions for Acute Exacerbations of COPD. Clinical Medicine Research. Vol. 2, No. 6, 2013, pp. 167-173. doi: 10.11648/j.cmr.20130206.20

\begin{abstract}
Background: Acute exacerbations of COPD (AECOPD) are known to be associated with increased morbidity and mortality and have a significant socioeconomic impact. The factors that determine frequent hospital readmissions for AECOPD are poorly understood. The aim of this study was to EVALUATE the factors responsible for the repeated admissions for AECOPD. Methodology: We conducted a prospective study of 235 patients with COPD with one or more admissions for acute exacerbations in a tertiary care hospital. Frequency of previous readmissions for AECOPD in the past year, and clinical characteristics, including spirometry were ascertained in the stable state both before discharge and at 6month post discharge. All the patients were followed up for a period of two years after discharge to evaluate the readmissions for the acute exacerbations. Results: Among 149 COPD patients who were admitted and assessed for AECOPD, there were 278 readmissions for AECOPD over a follow-up period of 24 months. Of these, $27 \%$ had one readmission, while rest $78 \%$ had two or more readmissions during this period. There was a high prevalence of current or ex-heavy smokers, associated co-morbidity, underweight patients and low prevalence of vaccination and use of domiciliary oxygen therapy among COPD patients. A total of $15.4 \%$ mortality was observed in the present study. The multivariate logistic regression analysis for repeated admissions revealed that disease duration $>10$ years $(\mathrm{OR}=0.50 ; 95 \% \mathrm{CI}$ : 0.27 $0.93)$, non-use of inhaled corticosteroids (ICS $+\mathrm{LABA})(\mathrm{OR}=2.31 ; 95 \% \mathrm{CI}$ : 2.26-4.88), lower serum albumin level $(<2 \mathrm{gm} / \mathrm{dl})(\mathrm{OR}=0.48 ; 95 \%$ CI: $0.26-0.88)$, MRC dyspnea grade $>3(\mathrm{OR}=1.15 ; 95 \%$ CI: $2.56-4.89)$, body mass index $(\mathrm{BMI})<20(\mathrm{OR}=0.62,95 \%$ CI: 0.31-1.23) and non-use of Tiotropium (OR $=1.21,95 \%$ CI: $0.67-2.19)$ were independently associated with frequent readmissions for AECOPD. Conclusion: The repeated exacerbations in COPD were common with major burden to the society. The major factors influencing frequency of repeated COPD exacerbations were disease duration, non-prescription of inhaled corticosteroids (ICS $+\mathrm{LABA})$ and Tiotropium, lower serum albumin level $(<2 \mathrm{gm} / \mathrm{dl})$, lower BMI and MRC dyspnea grade $>3$.
\end{abstract}

Keywords: COPD, Acute Exacerbation, AECOPD, Repeated Exacerbation, Hospitalization

\section{Introduction}

Chronic obstructive pulmonary disease (COPD) and acute exacerbations of COPD (AECOPD) have major impact both on the population and on health care costs. The burden of COPD and the impact of exacerbation on the health economy vary between countries and within regions of the same country, likely due to inconsistent to the differences in the risk factors for COPD. COPD morbidity and mortality, however, have increased over the past 40 years and this trend will continue, and it is projected to be the third leading cause of death by $2020^{1}$.

Exacerbations are common in COPD due to variety of reasons. Repeated exacerbations of COPD are associated with more protracted course of the disease, characterized by an accelerated decline in $\mathrm{FEV}_{1}$, decreased quality of life, persistent breathlessness, development of co-morbid diseases and premature mortality ${ }^{2}$. AECOPD requiring inpatient interventions are particularly important in that they incur a large cost, and each one has the potential for a fatal outcome ${ }^{3}$. The outcomes of medical treatment and care remain poor, with particularly high rates of hospital readmissions for AECOPD. Over half of COPD patients who are hospitalized for acute exacerbations are readmitted at least once in the ensuing 6 months $^{4}$. Hospitalization for AECOPD accounts for a large part of the high healthcare expenditure for COPD, estimated to be $40 \%$ of the total direct cost of medical care for COPD in the USA ${ }^{5}$. A better 
understanding of long-term outcome of COPD patients following hospitalization may help patients and their physicians to better manage the disease and will help in preventing repeated exacerbations.

The factors that determine repeated exacerbations in COPD are poorly understood. Factors that have been studied as predictors of mortality and other outcomes include FEV1, blood gases, co-morbidity, chronic mucus hypersecretion, muscle weakness, poor nutritional status, low BMI, socioeconomic status, number of previous physician visits or hospital admissions, influenza vaccination, pneumococcal vaccination, pulmonary rehabilitation, inhaled corticosteroids and long-term oxygen therapy ${ }^{6}$. However, these studies have produced inconsistent results with respect to the influence of FEV1, BMI and smoking ${ }^{7}$. Hence the present prospective study was done to evaluate the various risk factors associated for repeated hospitalizations for AECOPD.

\section{Materials and Methods}

\subsection{Study Design and Patient Selection}

This was a prospective study in a tertiary care hospital from January 2008 to December 2012. During the initial 36 months, the patients were included in the study. For the next 24 months all these patients were followed up systematically for stability of the disease or any adverse impact. Diagnosis of COPD was made according to the criteria set by GOLD $^{8}$. Severity of COPD condition was stratified based on post-bronchodilator $\mathrm{FEV}_{1}$ percent predicted using GOLD guidelines (Stage I - $\mathrm{FEV}_{1}>80 \%$; Stage II - FEV 1 50\%-80\%; Stage III -FEV $130 \%-50 \%$; and Stage IV $-\mathrm{FEV}_{1}<30 \%$ or $\mathrm{FEV}_{1}<50 \%$ along with signs of right ventricular failure). The inclusion criteria were: (i) patients with a principal diagnosis of AECOPD; (ii) age over 50 years; (iii) current smokers or ex-smokers with a history of smoking equivalent to at least 20 pack-years; and (iv) surviving patients with stable COPD status on discharge. Both COPD patients with first-episode exacerbation and repeated admissions were included. Patients with active pulmonary tuberculosis, pulmonary fibrosis, pneumothorax, pulmonary embolism, lung cancer, acute heart failure, acute myocardial infarction, stroke sequelae, severe renal failure, moderate to severe Parkinson's disease, dementia, hearing impairment, or those who were mentally unable were exclude from the study. An acute exacerbation was defined according to Anthonisen criteria ${ }^{2}$ : increased cough and sputum volume, increased sputum purulence and increased dyspnea. In addition patient may have had fever, malaise, fatigue and chest congestion. Severity of AECOPD was defined as Type I when patients had all 3 symptoms; Type II when patients had any two symptoms and Type III when patients had any one symptom. Each patient was treated in a standard fashion with $40 \quad-80 \quad \mathrm{mg}$ of parental methylprednisolone, nebulised bronchodilators (salbutamol and ipratropium bromide), theophyllines and supplemental oxygen. Antibiotics were used when there were signs of bacterial infection. In addition, some patients with respiratory failure were admitted to the ICU and started on mechanical ventilated, if required. All the patients were followed up for a period of 24 months after discharge for any future risk of exacerbations. If any fatality occurred during this period, the cause of death was ascertained as far as possible.

\subsection{Clinical Data}

Demographic and clinical data were collected for all patients including age, sex, socioeconomic and marital status, smoking load (pack-years), history of previous pneumonia, use of home oxygen, co-morbid conditions, detailed hemogram and biochemical, spirometric and arterial blood gas analysis at admission. In addition, admission to the ICU, length of hospital stay, BMI, duration of COPD, time elapsed since first hospitalization, use of daily medications including inhaled steroids, long acting beta 2 agonist (LABA), Ipratropium and tiotropium was also noted for each patient. Level of dyspnea was graded according to modified MMRC grading (Modified Medical Research Grading) from Grade 1 to Grade 4. Comorbid conditions were given scores according to Charlson to predict mortality. Each patient was followed up regularly for the next 24 months. The number of exacerbations that required_hospitalization over 24 months was recorded for each patient. In the event of death, cause of death was ascertained as far as possible either at home or in the hospital. Low BMI was used to categorize patients in two nutritional status groups: underweight $(<20 \mathrm{~kg} / \mathrm{m} 2)$ and normal or overweight $(\geq 20 \mathrm{~kg} / \mathrm{m} 2)$. Patient's level of treatment adherence was measured using a scale of three questions relating to the proportion of the times when the patient was able to take his medications as prescribed, attend follow-up and day-rehabilitation visits as required and undertake rehabilitative exercises as prescribed. Standard spirometric measurements at admission and during follow-up were performed using hand held minispirometer. The values of $\mathrm{FEV}_{1}$ and FVC were based upon the best of three readings. Written informed consent was taken from all the patients and the study was approved by the Institutional Ethical Review Board.

\subsection{Statistical Analysis}

All data was analyzed using the statistical package, SAS (version 6.12; SAS Inc., NC, USA). The distributions of normal variables were compared using the chi-squared test. Results were expressed as mean \pm SD for continuous variables or number and percentage for discrete variables. The prevalence of 'frequent' and 'non-frequent' readmissions and risk factors were expressed as numbers and percentages. Univariate analyses of risk factors that were associated with frequent readmissions were performed as for a case-control study, using the chi-square statistic and 
estimates of the odds ratio (OR) with their 95\% confidence intervals (CI). Multivariate analysis to evaluate the independent risk factors for frequent readmissions for AECOPD was performed using logistic regression for dichotomous dependent variable. Both forward stepwise selection and full saturated models were developed and used to evaluate estimates of ORs. A p value $<0.05$ was considered statistically significant.

\section{Results}

\subsection{Patient Baseline Characteristics}

A total of 235 patients with AECOPD over a period of 36 months were included in the study. Eighty six patients were dropped from the final analysis due to various reasons defaulted immediately after inclusion, left the residence, developed other co-morbidity, refused to give consent (Fig. 1). Thus a total of 149 patients were finally assessed. The mean age was $65.9 \pm 6.8$ years. A total of 278 exacerbations were observed over 24 months during the follow up period. Ninety-seven patients $(65.1 \%)$ were either current smokers or ex-smokers. Majority of patients $(81.5 \%)$ had stage II or stage III COPD disease. The incidence of co-morbidity varied significantly: diabetes (16.6\%), IHD (55.3\%), hypertension $(57.9 \%)$, renal disease $(16.3 \%)$, liver disease $(11.4 \%)$ and history of alcoholism (43.7\%). Nearly $30 \%$ had more than 2 co-morbid conditions (Table 1). About $65 \%$ of the patients in the present study were taking inhaled tiotropium, and another $55.5 \%$ were on the maintenance inhaled steroids along with beta ${ }_{2}$ agonists._Majority of the COPD patients had muscle wasting giving rise to low BMI and the mean serum albumin levels were $<3.00 \mathrm{gm} / \mathrm{dl}$.

The clinical characteristics of COPD patients with frequent and non-frequent readmissions are summarized in Table 2. Majority of the subjects were current smokers or ex-smokers with a history of smoking equivalent to at least 13 pack-years. Nearly 60 patients $(40 \%)$ had BMI $<20$ $\mathrm{kg} / \mathrm{m} 2$, another $74 \%$ patients had at least one co-morbid disease and 49 patients ( $32 \%)$ had $\geq 2$ co-morbid diseases. On multivariate analysis, MRC dyspnoea grade of $>3$ was associated with more chances of exacerbations than those with lesser grade of dyspnea (OR $=1.15, \mathrm{CI}: 2.56-4.89, \mathrm{p}<$ $0.001)$. Patients with long duration of COPD ( $>10$ years) were approximately two times more likely to have frequent readmissions than those with short duration $(\mathrm{OR}=0.50, \mathrm{CI}$ : $0.27-0.93, p<0.02)$. Severity of lung function did not correlated with the repeated exacerbation. Serum albumin levels $(<2 \mathrm{gm} / \mathrm{dl})$ and use of inhaled corticosteroids along

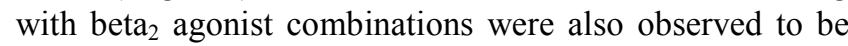
independent risk factors for the multiple admissions in patients with acute exacerbation of COPD $(\mathrm{OR}=0.48, \mathrm{CI}$ : $0.26-0.88, \mathrm{p}<0.01 ; \mathrm{OR}=2.21, \mathrm{CI}: 1.08-4.54, \mathrm{p}<0.02$ respectively). It was also observed that patients using inhaled corticosteroids along with long acting beta agonists developed less exacerbations than those patients who were not using this medication $(\mathrm{OR}=2.31$, CI: $2.26-4.88, \mathrm{p}<$
0.01). Similarly, patients on inhaled Tiotropium regularly during the follow-up period had good quality of life, and they had less development of repeated exacerbations COPD $(\mathrm{OR}=2.48$, CI: $1.26-3.28, \mathrm{p}<0.01)$. Very few of our patients received vaccinations including influenza and pneumococcal, hence the protective role of vaccination in these COPD patients cannot be commented upon. A total of 33 patients (22.1) died during the hospitalization either during the first episode or during the repeated exacerbations.

In the present study it was observed that 40 patients (26.8\%) had one exacerbation during the follow-up period of 2 years, while the remaining 109 patients $(73.2 \%)$ had more than one exacerbation during the follow-up period. We analyses the patients having one exacerbation as compared to those having more than one exacerbation during the follow-up period. On multivariate analysis, the independent risk factors observed for the repeated exacerbations were duration of COPD ( $>10$ years), MRC dyspnea grade $>3$, low serum albumin levels $(<2 \mathrm{mg} \%)$, low BMI $(<20)$ and limited use of ICS/LABA and Tiotropium for therapy (Table 3).

Table 1. Baseline characteristics of patients hospitalized for chronic obstructive pulmonary disease (COPD) exacerbations

\begin{tabular}{ll}
\hline Characteristics & Data, $\mathbf{n}=\mathbf{1 4 9} \mathbf{( \% )}$ \\
\hline Age (Years) & $65.9 \pm 6.8$ \\
Sex, Male & $105(70.4)$ \\
Smoking history & \\
Current/exsmoker & $97(65.1)$ \\
Non-smoker & $18(34.9)$ \\
Severity of lung disease & \\
Stage I & $5(3.3)$ \\
Stage II & $22(14.7)$ \\
Stage III & $72(48.3)$ \\
Stage IV & $50(33.6)$ \\
Maintenance inhaled steroids/LABA & $83(55.7)$ \\
Use of Tiotropium & $96(64.4)$ \\
History of previous pneumonia & $15(10.4)$ \\
Duration of disease & \\
$<10$ years & $25(16.7)$ \\
10- 19 years & $92(61.7)$ \\
$\geq 20$ years & $32(21.4)$ \\
Presence of pedal edema & $63(41.7)$ \\
BMI (kg/m $\left.{ }^{2}\right)$ & $18 \pm 3.5$ \\
Albumin g.dl ${ }^{-1}$ & $3.23 \pm 0.60$ \\
Comorbidity & \\
Diabetes & 88.9 \\
IHD & 100 \\
Hypertension & \\
Renal disease & $82(16.6)$ \\
Alcoholism & $86(55.3)$ \\
Liver disease & $24(16.3)$ \\
Treatments & $65(43.7)$ \\
Beta -agonists $_{\text {Anticholinergic agents }}$ & $16(11.4)$ \\
Inhaled corticosteroids & \\
Systemic corticosteroids & 97.2 \\
Oxygen therapy & 80.1 \\
Antibiotics & \\
\hline
\end{tabular}

* Data expressed as mean $\pm \mathrm{SD}$ or No. (\%). 


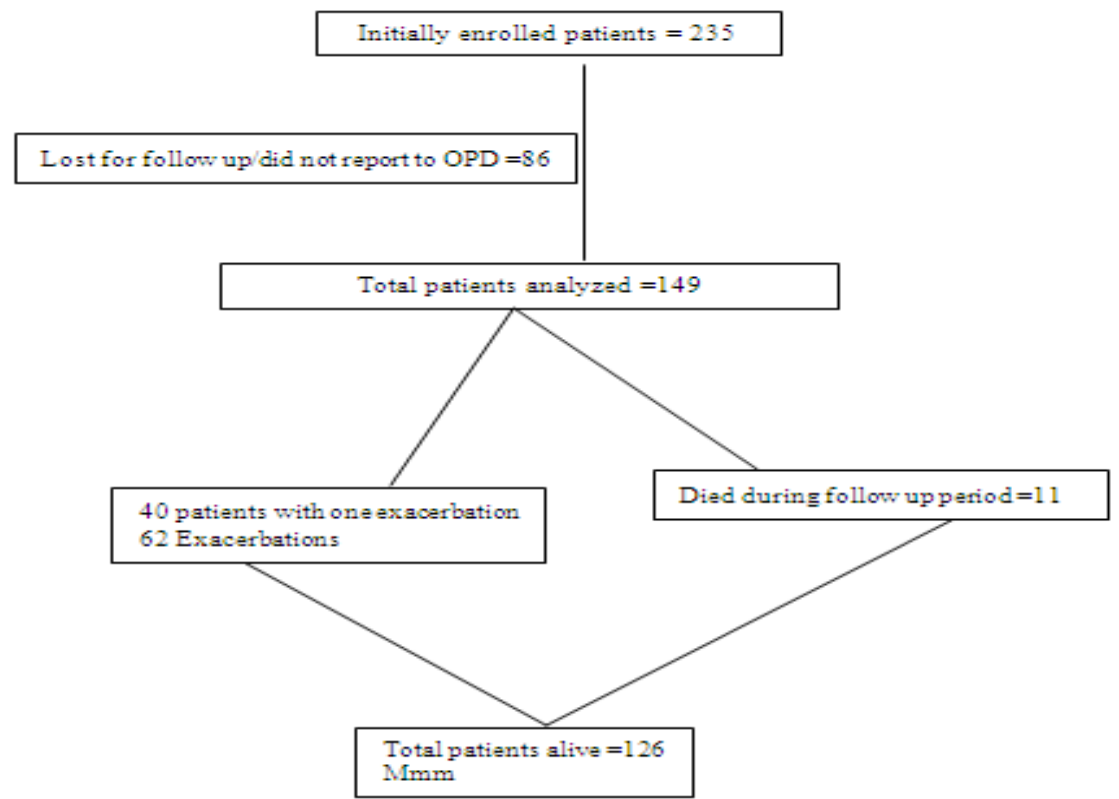

Fig 1. Distribution of all patients with exacerbations and failures.

Table 2. Multivariate risk factors for multiple readmissions to the hospital for AECOPD

\begin{tabular}{|c|c|c|c|c|c|}
\hline $\begin{array}{l}\text { Independent } \\
\text { Variables }\end{array}$ & $\begin{array}{l}\text { One admission } \\
\text { (40) }\end{array}$ & $\begin{array}{l}\text { Frequent } \\
\text { admissions } \\
\text { (75) }\end{array}$ & Odds Ratio & $95 \%$ confidence interval & p-value \\
\hline \multicolumn{6}{|l|}{ BMI $\left(\mathrm{kg} / \mathrm{m}^{2}\right)$} \\
\hline$>20$ & 42 & 17 & 1.40 & $0.76-2.61$ & 0.27 \\
\hline$<20$ & 20 & 158 & 0.62 & $0.31-1.23$ & 0.001 \\
\hline Current smoker & 13 & 51 & 0.51 & $0.23-1.13$ & 0.10 \\
\hline \multicolumn{6}{|c|}{ MRC Dyspnea Grading } \\
\hline$<3$ & 35 & 16 & 0.68 & $0.38-1.21$ & 0.19 \\
\hline$>3$ & 27 & 59 & 1.15 & $2.56-4.89$ & $<0.001$ \\
\hline $\mathrm{FEV}_{1}<50 \%$ pred & 45 & 59 & 0.77 & $0.44-1.33$ & 0.34 \\
\hline \multicolumn{6}{|l|}{ Duration of COPD } \\
\hline$>10$ years & 26 & 62 & 0.50 & $0.27-0.93$ & $<0.02<10$ \\
\hline years & 24 & 13 & 1.54 & $0.71-3.34$ & 0.10 \\
\hline \multicolumn{6}{|c|}{ No. of co-morbidities } \\
\hline$>2$ & 35 & 49 & 0.73 & $0.40-1.37$ & 0.29 \\
\hline Use of Tiotropium & 21 & 51 & 1.21 & $0.67-2.19$ & 0.001 \\
\hline Use of ICS/LABA & 37 & 46 & 2.31 & $2.26-4.88$ & $<0.01$ Serum \\
\hline albumin & 26 & 45 & 0.48 & $0.26-0.88$ & $<0.01(<2 \mathrm{gm} / \mathrm{dl})$ \\
\hline
\end{tabular}

Table 3. Significant factors associated with frequent readmissions in COPD (results of forward stepwise selection multiple logistic regression)

\begin{tabular}{lccc}
\hline \multicolumn{3}{c}{ Significant independent } \\
Predictors & Odds ratio & $\mathbf{9 5 \%}$ CI & P-value \\
\hline Body mass Index $<20$ & 0.62 & $0.31-1.23$ & 0.001 \\
MRC Dyspnea Grade $>3$ & 1.15 & $2.56-4.89$ & $<0.001$ \\
Duration of COPD $>10$ years & 0.50 & $0.27-0.93$ & $<0.02$ \\
Serum albumin $<2.0 \mathrm{mg} \%$ & 0.48 & $0.26-0.88$ & $<0.01$ \\
Use of ICS/LABA & 2.31 & $2.26-4.88$ & $<0.01$ \\
Use of Tiotropium & 1.21 & $0.67-2.19$ & 0.001 \\
\hline
\end{tabular}

\section{Discussion}

Exacerbations in COPD are common and they are known to be associated with increased morbidity and mortality with a significant socioeconomic impact. Patients with frequent exacerbations often experience impaired quality of life and a faster decline in lung function over time. Severe exacerbations that lead to hospitalization constitute a risk for mortality both during the hospitalization and during the subsequent years. In the present study we have tried to analyze various factors leading to frequent hospitalizations for AECOPD.

The present study describes the outcome of 149 patients 
who experienced at least one AECOPD resulting in hospitalization over a 36 months period. All the patients were followed-up for the next 24 months. During the study period, the mean duration from the index admission to the first readmission was only $3 \pm 4.08$ months. Failure was considered if the patient was readmitted within 30 days after discharge. The failure rate in the present study was observed to be $34.8 \%$. A previously described cohort of 345 COPD patients ${ }^{9}$ admitted for AECOPD observed a similar failure rate after single exacerbation. The authors reported a first readmission rate of $63 \%$ over a mean follow-up period of 1.1 years and a median time to first readmission of 186 days. Bahodori et $\mathrm{al}^{10}$ has also reported high failure rates after discharge following AECOPD. The high frequency of readmission and the short time to readmission found in all these studies, highlight the social and economic burden that COPD exerts on society. In the study done by Dewan et $\mathrm{al}^{11}$, treatment failure was observed in $12.1 \%$ of first exacerbations and $14.7 \%$ of all exacerbations, with more than half the failures requiring hospitalization. The various factors that were independently associated with treatment failure included $\mathrm{FEV}_{1}<35 \%$, use of home oxygen, frequency of exacerbation, history of previous pneumonia, history of sinusitis and use of maintenance steroids.

Risk factors for exacerbations in COPD are multifactorial, with the predominant being respiratory infections and air pollution ${ }^{12}$. COPD patients also have frequent comorbid conditions, particularly coexistent cardiac disease, hypertension, diabetes, etc. and they have been shown to be a risk factor for increased hospital admission and mortality in patients with AECOPD $^{13,14}$. Patients with moderate to severe COPD patients are more likely to have cardiovascular disease. In the present study, almost 51\% had associated cardiac disease. Elevated pulmonary artery pressure can lead to right heart failure and ultimately death. It has been observed in various studies that pulmonary artery pressure $>18 \mathrm{mmHg}$ doubled the risk of subsequent COPD hospitalizations ${ }^{15,16}$. In addition to the elevated pulmonary artery pressure, a common finding associated with COPD is diastolic dysfunction. In a retrospective study ${ }^{17}$, it was found that patients with diastolic dysfunction had 1.28 exacerbations compared with 0.67 in the control group. Diabetes mellitus, which is found more frequently in COPD, may also be a risk factor for AECOPD ${ }^{9}$. Elevated blood sugars can increase the growth of pathogens in the airway as well as promote inflammation and predispose to the exacerbations ${ }^{18}$. In large unselected populations of COPD patients, it has been observed that half of those patients who are hospitalized are expected to be readmitted at least once in the ensuing 6 months with a majority $(86 \%)$ of readmissions occurring within the first 3 months after hospital discharge ${ }^{19,20}$.

In the present study, despite evaluating a wide range of both modifiable and non-modifiable potential risk factors for hospital readmissions, only a few clinical factors were found to be independently associated with COPD readmissions. The factors that were associated with repeated AECOPD were disease duration, use of inhaled corticosteroids (ICS +LABA), lower albumin levels, lower BMI and MRC dyspnea grade $>3$. It has been observed that home oxygen as a risk factor associated with an increased risk of admission or frequent readmissions ${ }^{21}$. It is likely that home $\mathrm{O} 2$ use is a marker of disease severity, and individuals with more advanced disease are at higher risk for readmission. In the present study very few patients were using long term oxygen therapy, hence the direct conclusions becomes difficult to arrive in present scenario. Previous hospitalization is a strong risk factor for repeated exacerbation in COPD patients $(\mathrm{p}<0.0001)$. The frequency and proximity of previous exacerbations are important predictors of subsequent exacerbations and hospitalizations. Maintaining muscle mass and activity is another critical aspect in the management of AECOPD. Some studies have shown increased risk for AECOPD in patients with low BMI and lower albumin levels ${ }^{22,23}$. In the present study also, lower albumin level $(<2 \mathrm{gm} / \mathrm{dl})$ was an independent risk factor for AECOPD $(\mathrm{p}<0.001)$.

As the severity of COPD increases, the likelihood of admission during exacerbations increases. In the present study the patients having disease for $>10$ years had more relapses as compared to shorter duration of the disease. Advanced COPD disease is associated with frequent exacerbation; Stage IV disease had frequent exacerbations as compared to mild to moderate form of disease $(p<0.04)$. Bahadosi et $\mathrm{al}^{10}$ did not observe any relation between $\mathrm{FEV}_{1} \%$ predicted and the rates of COPD readmissions. Several studies have similarly reported a lack of association between $\mathrm{FEV}_{1} \%$ predicted and COPD exacerbations and admissions $^{24}$; however, others ${ }^{25}$ have found lower $\mathrm{FEV}_{1}$ to be predictive of a higher risk of COPD admissions. The lack of association between $\mathrm{FEV}_{1}$ and hospital readmission may be due to relatively limited variation in the $\mathrm{FEV}_{1}$ across the study population ${ }^{25}$. Previous studies ${ }^{26}$ have also indicated that increased disease severity is associated with prolonged length of stay (LOS). In the present study mean LOS was about $8 \pm 4$ days. It may be that, shorter LOS was an indication of premature discharge before the clinical and social aspects of the admission were fully resolved and, thus, patients were more likely to return to hospital.

In the present study, on multivariate analysis, it was observed that use of Tiotropium is an important risk factor in preventing relapse due to AECOPD ( $\mathrm{p}<0.001)$ (OR 1.21; CI:0.67-2.19

After final analysis, it was also observed that combination of inhaled corticosteroids and long acting beta agonist (ICS + LABA) had lower relapse rates due to AECOPD $(\mathrm{p}<0.02)((\mathrm{OR}=2.31, \mathrm{CI}: 2.26-4.88, \mathrm{p}<0.01)$. More recent studies ${ }^{27,28}$ have demonstrated the effect of tiotropium on the reduction in the frequency of exacerbations. In one study ${ }^{27}$ the frequency of exacerbations was reduced with tiotropium from 2.41 per year in the placebo group to 1.57 in the tiotropium group ( $p$ $<0.001$ ). UPLIFT trial ${ }^{28}$, which included 5993 patients, it was observed that tiotropium significantly delayed time-to- 
first exacerbation (16.7 versus 12.5 months) and time-tofirst hospitalization for exacerbations ( HR 0.86; 95\% CI $0.78-0.95 ; \mathrm{p}<0.002$ ). Tiotropium also reduced the mean number of exacerbations by $14 \%(p<0.001)$. The addition of a long-acting bronchodilator and ICS (FSC) (salmeterol and fluticasone) in the same inhaler represents advancement and has recently been studied in TORCH study $^{29}$. The results from the study demonstrated a reduction in mortality of $2.6 \%$ with FSC in absolute terms compared to placebo, representing a relative mortality reduction of $17.5 \%(\mathrm{p}<0.05)$. It was also observed that FSC reduced the frequency of moderate or severe exacerbations significantly by $25 \%$ as well as hospitalizations by $17 \%$. In patients with stage II COPD, FSC reduced the frequency of exacerbations by $31 \%$. In the present study also FSC combination reduced the number of AECOPD.

The most profound effect of AECOPD is on mortality. The mortality in the present study was about $22 \%$. SolerCataluna $^{30}$ showed that AECOPD increases mortality with repeated admissions. Factors contributing to increased mortality are metastatic cancer, weight loss, pulmonary hypertension, heart failure, male gender, and previous COPD exacerbation hospitalizations ${ }^{14}$. Fruchter and Yiegla $^{31}$ evaluated the predictors for long term survival in patients with AECOPD, and they observed in-hospital mortality rate of $7.25 \%$. The risk of mortality at 1,3 and 5 years was $28 \%, 47 \%$ and $54 \%$, respectively. In multivariate analysis, current smoking, ischemic heart disease, $\mathrm{PaCO}_{2}$ on admission, hospital readmissions and $\mathrm{FEV}_{1}$ were independent predictors of mortality. It has been observed that if patients need intensive care or mechanical ventilation during a COPD exacerbation, they will usually have a protracted hospital stay and poor hospital outcomes $^{32}$.

After the discharge following AECOPD, regular follow up of these COPD patients was maintained for the next 2 years. It was observed that there was neither improvement in the grade of dyspnea nor improvement in BMI during the follow up period. COPD exacerbations have a negative effect on quality of life in patients with COPD. The quality of life is impaired not only in patients who are hospitalized for AECOPD, but also in patients who experience frequent exacerbations that are treated in the ambulatory care setting. It has been observed that repeated exacerbations have impact on health-related quality-of-life and it has been demonstrated that interventions that reduce exacerbations are associated with improvements in quality of life $\mathrm{e}^{32}$. It has been observed by Anzueto et $\mathrm{al}^{33}$ that about $60 \%$ of the patients survived for a median of 2 years after hospitalization for AECOPD, but their quality of life was poor. Many factors, such as air flow limitation, comorbidity, frequency of COPD exacerbations, and activities of daily living can have impact on health related quality of life in COPD patients.

\section{Summary}

Frequent admissions due to acute exacerbations are common in COPD, and various factors contribute for this condition. Long disease duration, low BMI, severe dyspnea grade, low prescription of inhaled Tiotropium and ICS/LABA were independently associated with frequent hospital readmissions for acute exacerbation in COPD patients. The low prevalence of caregiver support, pulmonary rehabilitation and vaccination and the high prevalence of current or ex-heavy smokers indicate that this disease remains poorly understood and an inadequately managed health problem. These results raise the possibility that these variables could be used as identifiers of patients at highest risk for frequent exacerbations and frequent hospitalizations for AECOPD.

\section{References}

[1] Rabe KF, Hurd S, Anzueto A (2007). Global Initiative for Chronic Obstructive Lung Disease, et al. Global strategy for the diagnosis, management, and prevention of chronic obstructive pulmonary disease: GOLD executive summary. Am J Respir Crit Care Med 176:532-555.

[2] Houlguin F, Folch E, Redd SC, et al (2005). Mannino DM. Comorbidity and mortality in COPD-related hospitalizations in the United States, 1979 to 2001. Chest 128:2005-2011.

[3] Mnanino DM, Buist AS (2007). Global burden of COPD: risk factors, prevalence, and future trends. Lancet 370:765773 .

[4] Connors AF, Dawson NV, Thomas C et al (1996). Outcomes following acute exacerbation of severe chronic obstructive lung disease. The SUPPORT investigators (Study to Understand Prognoses and Preferences for Outcomes and Risks of Treatments). Am J Respir Crit Care Med. 154: 959-67.

[5] National Institutes of Health (2002). National Heart, Lung and Blood Institute. Morbidity and Mortality: 2002 Chart Book on Cardiovascular, Lung and Blood Diseases. May 2002. [Cited 5 September 2003] Available from URL: http://www.nhlbi.nih.gov (accessed on October $\left.12^{\text {th }}, 2013\right)$.

[6] Garcia-Aymerich J, Farrero E, Félez MA, et al (2003). Risk factors of re-admission to hospital for a COPD exacerbation: a prospective study. Thorax 58: 100-5.

[7] Pouw EM, Ten Velde GP, Croonen BH, at al (2000). Kester $\mathrm{AD}$, Schols AH, Wouters EF. Early non-elective readmission for chronic obstructive pulmonary disease is associated with weight loss. Clin Nutr 19: 95-9.

[8] Rodriguez-Roisin R, Anzueto A, Bourbeau J, at al (2010). Global Initiative for Chronic Obstructive Lung Disease. Global strategy for the diagnosis, management, and prevention of chronic obstructive pulmonary disease: GOLD executive summary (Updated 2010). 1-117.

[9] Groenewegen KH, Schols AM, Wouters EF (2003). Mortality and mortality-related factors after hospitalization for acute exacerbation of COPD. Chest 124:459-467. 
[10] Bahadori K, FitzGerald JM, Levy RD, at al (2009). Risk factors and outcomes associated with chronic obstructive pulmonary disease exacerbations requiring hospitalization. Can Respir J 16(4):e43-e49.

[11] Wang Q, Bourbeau J (2005). Outcomes and health-related quality of life following hospitalization for an acute exacerbation of COPD. Respirology 10:334-340.

[12] Celli BR (1997). Is pulmonary rehabilitation an effective treatment for chronic obstructive pulmonary disease? Yes. Am J Respir Crit Care Med 155: 781-3.

[13] Nichols KL, Baken L, Nelson A (1999). Relation between influenza vaccination and outpatient visits, hospitalization, and mortality in elderly persons with chronic lung disease. Ann Intern Med 130: 397-403.

[14] Vestbo J, Prescott E, Lange P et al (1998). Vital prognosis after hospitalization for COPD: a study of a random population sample. Respir Med 92: 772-776.

[15] Advisory Committee on Immunization Practices (ACIP) (1998). Prevention and control of influenza: recommendations of the advisory committee on immunization practices (ACIP). MMWR Morb Mortal Wkly Rep 47: 1-26.

[16] Kesseler R, Faller M, Weitzenblum E et al (2001). "Natural history" of pulmonary hypertension in a series of 131 patients with chronic obstructive lung disease. Am J Respir Crit Care Med 164:219-224.

[17] McGhan R, Radcliff T, Fish R et al (2009). Predictors of rehospitalization and death after a severe exacerbation of COPD. Lung 187:128-135.

[18] Abusaid GH, Barbagelata A, Tuero E et al (2009). Diastolic dysfunction and COPD exacerbation. Postgrad Med 121:7681.

[19] Douglas SL, Daly BJ, Gordon N et al (2002). Survival and quality of life: short-term versus long-term ventilator patients. Crit Care Med 30: 2655-62.

[20] Baker EH, Janaway CH, Philips BJ et al (2006). Hyperglecemia is associated with poor outcomes in patients admitted to hospital with acute exacerbation of chronic obstructive pulmonary disease. Thorax 61:284-289.

[21] Garcia-Aymerich J, Barreiro E, Farrero E et al (2000). Patients hospitalized for COPD have a high prevalence of modifiable risk factors for exacerbation (EFRAM study). Eur Respir J 16: 1037-1042.
[22] Horn SD, Sharkey PD, Buckle JM et al (1991). The relationship between severity of illness and hospital length of stay and mortality. Med Care 29:305-317.

[23] Skyba P, Kluchova Z, Joppa P ET AL (2009). Nutritional status in relation to respiratory impairment and systemic inflammation in patients with acute exacerbation of COPD. Med Sci Mon 15:CR528-CR533.

[24] Tsimogianni AM, Papiris SA, Stathopoulos GT ET AL (2009). Predictors of outcome after exacerbation of chronic obstructive pulmonary disease J Gen Intern Med 24:10431048.

[25] Emerman CL, Effron D, Lukens TW (1991). Spirometric criteria for hospital admission of patients with acute exacerbation of COPD. Chest 99:595-599.

[26] Seemungal TA, Donaldson GC, Paul EA et al (1998). Effect of exacerbation on quality of life in patients with chronic obstructive pulmonary disease. Am J Respir Crit Care Med 157(5 Pt 1):1418-1422.

[27] Dusser D, Bravo M-L, Iacono P (2006). The effect of tiotropium on exacerbations and airflow in patients with COPD. Eur Respir J 27: 547-555.

[28] McGhan R, Radcliff T, Fish R et al (2007). Predictors of rehospitalization and death after a severe exacerbation of COPD. Chest 132:1748-1755.

[29] Tashkin DP, Celli B, Senn S et al (2008). A 4-year trial of tiotropium in chronic obstructive pulmonary disease. $\mathrm{N} \mathrm{Engl}$ J Med 359: 1543-1554.

[30] Calverley PMA, Anderson JA, Celli B et al (2007). Salmeterol and fluticasone propionate and survival in chronic obstructive pulmonary disease. N Engl J Med 2007; 356: 775-789.

[31] Soler-Catalufia JJ, Martinez-Garcia MA, Roman Sanchez E et al (2005). Severe acute exacerbations and mortality in patients with chronic obstructive pulmonary disease. Thorax 60:925-931

[32] Fruchter O, Yigla M (2008). Predictors of long-term survival in elderly patients hospitalized for acute exacerbations of chronic obstructive pulmonary disease. Respirology 13:851855 .

[33] Wang Q, Bourbeau J (2005). Outcomes and health-related quality of life following hospitalization for an acute exacerbation of COPD. Respirology 10:334-340. 